\title{
Low-sugar yogurt making by the co-cultivation of Lactobacillus plantarum WCFS1 with yogurt starter cultures
}

\author{
S. S. Zhang, ${ }^{1}$ Z. S. $X u{ }^{2}$ L. H. Qin, ${ }^{1}$ and J. Kong ${ }^{1 *}$ (]) \\ ${ }^{1}$ State Key Laboratory of Microbial Technology, Shandong University, Qingdao, 266237, P. R. China \\ ${ }^{2}$ State Key Laboratory of Biobased Material and Green Papermaking, Qilu University of Technology, Shandong Academy of Science, Jinan, \\ 250353, P. R. China
}

\section{ABSTRACT}

Yogurt making traditionally relies on the simultaneous utilization of 2 starters: Streptococcus thermophilus and Lactobacillus bulgaricus, but these 2 strains normally metabolize the glucose portion of lactose and release galactose into extracellular medium, resulting in high levels of residual galactose and unfermented lactose in yogurt, which leads to several industrial and health concerns. In this study, we found that Lactobacillus plantarum could effectively metabolize both lactose and galactose. Comparative genomic analysis demonstrated the constant presence of a chromosomeencoded Leloir pathway for galactose metabolism in $L b$. plantarum species, and the gal operon was driven by a strong constitutive promoter in Lb. plantarum WCFS1, displaying great potential in low-sugar yogurt making. To test this hypothesis, Lb. plantarum WCFS1 was co-cultured with S. thermophilus or Lb. bulgaricus in lactose-based medium. Results showed that lactose was consumed completely and galactose was metabolized efficiently. For yogurt making, co-cultivation of $L b$. plantarum WCFS1 with yogurt starter cultures produced a higher reduction of total sugar content compared with the traditional fermentation processes. In addition, the sensory analysis indicated that the yogurt fermented with yogurt starter cultures and $L b$. plantarum WCFS1 was acceptable to consumers in appearance, texture, and flavor. Therefore, this study emphasized the potential to manufacture low-sugar yogurt by the co-cultivation of $L b$. plantarum with yogurt starter cultures.

Key words: Lactobacillus plantarum, low-sugar yogurt, traditional starter, Leloir pathway

\section{INTRODUCTION}

Yogurt is one of the most popular fermented dairy products and has high consumer acceptability world-

Received July 28, 2019.

Accepted December 17, 2019.

*Corresponding author: kongjian@sdu.edu.cn wide for its high nutritional value, unique flavor, and health benefits (Benozzi et al., 2015; Aryana and Olson, 2017). Yogurt is traditionally produced by the cultivation of Streptococcus thermophilus and Lactobacillus delbrueckii ssp. bulgaricus (Horiuchi et al., 2009). However, most strains of $S$. thermophilus and Lb. bulgaricus only metabolize the glucose moiety of lactose and release the galactose into the medium, resulting in significant amounts of galactose as well as high levels of residual unfermented lactose in yogurt (Turner and Martley, 1983; Hols et al., 2005). Lactose and galactose are not recommended for individuals with symptoms of lactose intolerance and galactosemia (Van Calcar et al., 2014; Silanikove et al., 2015). In addition, residual galactose in fermented dairy products was associated with several industrial problems such as reduced quality of fermented products, browning in pizza, and textural defects in cheese (Neves et al., 2010; Wu et al., 2015). Therefore, efforts to remove or reduce lactose and galactose are of great importance to human health and industrial processes (Chollet et al., 2013).

Lactase is commonly used to hydrolyze lactose into galactose and glucose to produce lactose-free milk (Jelen and Tossavainen, 2003; Harju et al., 2012). This method is expensive and cannot eliminate the galactose from milk. The obtained dairy foods are only suitable for individuals with lactose intolerance and not for the patients with galactosemia. In this case, various chromatographic methods for the separation of lactose from other milk constituents were carried out, which can eventually produce lactose-free and galactose-free milk products (Harju et al., 2012). However, these commercial lactose-free dairy products are sold in unfermented status. Considering the nutrition and health-promoting benefits of yogurt compared with nonfermented dairy products, research has been done on isolating or constructing wild-type or modified $S$. thermophilus strains with galactose-positive $\left(\mathrm{Gal}^{+}\right)$phenotype, and then coculturing with $L b$. bulgaricus for the preparation of low-galactose yogurt (Robitaille et al., 2007; Anbukkarasi et al., 2014a). However, the modified strain only metabolized galactose when the amount of lactose was 
less than $0.1 \%$ (wt/vol), and the $\mathrm{Gal}^{+}$phenotype was not expressed during milk fermentation and mozzarella cheese production (Robitaille et al., 2007). Moreover, there is a ceiling on lactose hydrolysis when $S$. thermophilus and $L b$. bulgaricus grow in milk. Therefore, an alternative method is needed to manufacture lactosefree or galactose-free yogurt.

Lactobacillus plantarum is a versatile facultative hetero-fermentative lactic acid bacterium present in plant-based fermented foods as well as meat, fish, and fermented dairy products (Marco et al., 2017). Its dual role as an indigenous human gut inhabitant and a safe starter culture in food fermentation makes it a preferred candidate for the development of novel functional products (Capozzi et al., 2017). Several studies have confirmed that consuming $L b$. plantarumcontaining fermented milk brought in vivo functionality to host health (Chiu et al., 2006; Valente et al., 2019). However, the effects of Lb. plantarum on residual lactose or galactose in fermented dairy foods have not been reported yet. In our previous studies, 2 functional $\beta$-galactosidase genes responsible for lactose hydrolysis were identified in the genome of $L b$. plantarum WCFS1. Here, we investigated the genetic organization of the lactose hydrolysis and galactose metabolism in $L b$. plantarum by comparative genome analysis, and determined the residual sugar content in the medium when Lb. plantarum WCFS1 was inoculated alone or cocultured with traditional yogurt starters. The objective of this study was to determine whether the content of lactose or galactose could be decreased by the addition of $L b$. plantarum, as well as its effect on the properties of dairy products.

\section{MATERIALS AND METHODS}

\section{Bacterial Strains, Plasmids, and Cultivation Conditions}

Bacterial strains and plasmids used in this study are listed in Supplemental Table S1 (https://doi.org/ 10.3168/jds.2019-17347). As the host for plasmid construction, Lactococcus lactis MG1363 was grown statically in M17 broth (Oxoid, Basingstoke, UK) supplemented with $0.5 \%$ (wt/vol) glucose (GM17) at $30^{\circ} \mathrm{C}$. Streptococcus thermophilus CGMCC 7.179 was a $\mathrm{Gal}^{+}$ strain and SDMCC050215 was a $\mathrm{Gal}^{-}$strain, and they were grown statically in M17 broth supplemented with $1 \%$ (wt/vol) lactose (LM17) at $42^{\circ} \mathrm{C}$, and the strains of Lb. plantarum and Lb. bulgaricus were grown statically in de Man, Rogosa, and Sharpe (MRS) medium (Oxoid, Basingstoke, UK) at $37^{\circ} \mathrm{C}$. The M17-galactose broth was prepared by supplemented with $1 \%$ (wt/ vol) galactose in M17 broth. The MRS-lactose or MRSgalactose broth was prepared by substitution of glucose with 1\% (wt/vol) lactose or galactose in MRS medium, respectively. If necessary, chloramphenicol with a final concentration of $5 \mu \mathrm{g} / \mathrm{mL}$ was added to the medium for Lc. lactis or Lb. plantarum recombinant strains.

\section{Growth and Metabolism of Lb. plantarum WCFS1 and Traditional Starters in Lactose/ Galactose-Based Medium}

The MRS-lactose and MRS-galactose medium were used to test the ability of $L b$. plantarum WCFS1 and $L b$. bulgaricus to use lactose and galactose. The LM17 and M17-galactose medium were used to test the ability of $S$. thermophilus to use lactose and galactose. After overnight static incubation, Lb. plantarum WCFS1 and Lb. bulgaricus (ATCC11842 and SDMCC050201) were inoculated $(2 \% \mathrm{vol} / \mathrm{vol})$ into the MRS-lactose and MRS-galactose medium at $37^{\circ} \mathrm{C}$, S. thermophilus (CGMCC 7.179 and SDMCC050215) were inoculated ( $2 \% \mathrm{vol} / \mathrm{vol})$ into the LM17 and M17-galactose medium at $42^{\circ} \mathrm{C}$. Samples of the cultures were collected at interval of $2 \mathrm{~h}$ for analysis during a period of $24 \mathrm{~h}$. The growth was measured by optical density at $600 \mathrm{~nm}$ $\left(\mathbf{O D}_{600}\right)$ with Biophotometer. The $\mathrm{pH}$ was measured with a glass electrode $\mathrm{pH}$ meter (Mettler Toledo, $\mathrm{Zu}$ rich, Switzerland). The content of residual sugars and metabolites in the cultures were determined by HPLC as described previously (Xu et al., 2017).

\section{Molecular Manipulation}

All the molecular manipulations in this study were performed as described previously (Xu et al., 2018). Ex Taq polymerase, restriction enzymes, and $\mathrm{T}_{4} \mathrm{DNA}$ ligase were used according to manual protocols (TaKaRa, Tokyo, Japan). Genomic DNA of Lb. plantarum WCFS1 was extracted using the Bacterial Genomic Extraction Kit (Tiangen, Beijing, China). Primers Pgal-F (5'-CGCAGATCTGAGGAAGCTCCTTTCAAAGT-3') and Pgal-R (5'-CGCGAATTCCATGAAAAATCCCTCCGTAAATAG-3') were designed for amplification of the gal operon promoter from the genomic DNA of $L b$. plantarum WCFS1. The PCR product was digested with $B g l I I$ and $E c o R I$ and cloned into the corresponding sites of plasmid pSec:Leiss:Nuc, resulting in recombinant plasmid pGal. Primers GFP-F (5'-CGCGAATTCAGCAAAGGAGAAGAACTTTT- $\left.3^{\prime}\right)$ and GFP-R (5'-GCGGATATCTTAGTATAGCTCATCCATGC-3') were used for amplification of the reporter gene $g f p$ using pCD4033-gfp as the template (Sun et al., 2010). The PCR product was digested with EcoRI and EcoRV and 
ligated into the corresponding sites of plasmid pGal, generating the recombinant plasmid pGal-GFP.

\section{Fluorescence Analysis}

Plasmid pGal-GFP was transformed into Lb. plantarum WCFS1, generating recombinant $L b$. plantarum WCFS1/gal-GFP. This strain was grown statically in the MRS-glucose, MRS-lactose, and MRS-galactose media at $37^{\circ} \mathrm{C}$ for $12 \mathrm{~h}$. Cells were harvested by centrifugation at $6,000 \times g$ for $5 \mathrm{~min}$ at $4^{\circ} \mathrm{C}$, washed twice, and resuspended in PBS buffer $(137 \mathrm{mM} \mathrm{NaCl}, 2.7$ $\mathrm{m} M \mathrm{KCl}, 10 \mathrm{~m} M \mathrm{Na}_{2} \mathrm{HPO}_{4}$, and $2 \mathrm{mM} \mathrm{KH}_{2} \mathrm{PO}_{4}$, pH 7.4). For measurement of fluorescence intensity, 200 $\mu \mathrm{L}$ of bacterial suspension was transferred into a 96well plate, and the Multi-Detection Microplate Reader (BioTek, Winooski, VT) was used by excitation at $485 \mathrm{~nm}$ and emission at $535 \mathrm{~nm}$. The empty vectorcontaining strain was used as control in each respective medium.

\section{Coculture of Lb. plantarum with Traditional Starters in Lactose-Based Medium}

Streptococcus thermophilus strains (CGMCC 7.179 and SDMCC050215) and Lb. bulgaricus strains (ATCC11842 and SDMCC050201) were used to test the utilization of lactose and galactose with or without Lb. plantarum WCFS1. The cultures of $S$. thermophilus were inoculated $(2 \% \mathrm{vol} / \mathrm{vol})$ to the LM17 broth at $42^{\circ} \mathrm{C}$ and $L b$. bulgaricus were inoculated $(2 \% \mathrm{vol} / \mathrm{vol})$ to the MRS-lactose broth at $37^{\circ} \mathrm{C}$. When co-cultured, $2 \%$ (vol/vol) Lb. plantarum WCFS1 was added. Samples of the cultures were collected at interval of $2 \mathrm{~h}$ for analysis during a period of $24 \mathrm{~h}$. The growth was measured by $\mathrm{OD}_{600}$ and viable cell counts. The $\mathrm{pH}$ was measured with a glass electrode $\mathrm{pH}$ meter. The sugars and lactic acid were measured by HPLC as described above.

\section{Fermented Milk}

Reconstituted skim milk (RSM) medium was prepared by reconstituting skim milk powder $(10 \% \mathrm{wt} /$ vol) with distilled water and sterilized at $121^{\circ} \mathrm{C}$ for 15 min. Three strains (S. thermophilus, Lb. bulgaricus, and Lb. plantarum WCFS1) were co-cultured in the ratio of 1:1:1 in $100 \mathrm{~mL}$ of RSM medium at $42^{\circ} \mathrm{C}$ for $12 \mathrm{~h}$, and the fermentation without $L b$. plantarum WCFS1 was used as control. The curd time was observed visually, which indicated the time when milk changed from a flowing liquid to an immobile semi-solid state. At the end of the fermentation, the samples were cooled in an ice-water bath and stored at $4^{\circ} \mathrm{C}$ for $14 \mathrm{~d}$. The determi- nations of lactose and galactose content, $\mathrm{pH}$, titratable acidity, and syneresis were carried out at the end of fermentation, and the viable cell counts were detected at 0,7 , and $14 \mathrm{~d}$ as described by da Costa et al. (2016) and Li et al. (2017).

\section{Sensory Analysis}

Thirty panelists were selected and invited to take part in the sensory analysis. The yogurt samples were served in 20-mL plastic cups coded with random digit numbers at approximately $10^{\circ} \mathrm{C}$. Water was provided between evaluations of samples for mouth rinsing. Sample acceptance including the appearance, texture, and flavor was rated using a 10-point hybrid hedonic scale, where $1=$ disliked extremely and $10=$ liked extremely. Overall mean score was calculated for each sample by averaging the score of all participants.

\section{Statistical Analysis}

Each experiment was carried out in triplicate, and the data were presented as mean \pm standard deviation. Statistical significance between yogurts fermented by traditional starters with or without $L b$. plantarum WCFS1 was performed using the Statistical Packages for the Social Sciences (SPSS version 19.0, IBM Corp., Armonk, NY). Probability values of $<0.05$ were considered as statistically significant.

\section{RESULTS}

\section{Utilization of Lactose and Galactose by Lb. plantarum WCFS1}

Lactobacillus plantarum WCFS1 was cultivated for $24 \mathrm{~h}$ in the medium using lactose as the sole carbon source. Results showed that $99 \%$ lactose was consumed, galactose was almost undetectable, and a high level of lactic acid was produced, which indicated that $L b$. plantarum WCFS1 could metabolize lactose effectively and consume galactose at the same time (Figure 1A). To further confirm the ability of $L b$. plantarum WCFS1 to use galactose, it was cultivated in the MRS-galactose medium. The results indicated that $L b$. plantarum WCFS1 could consume $94 \%$ galactose after $24 \mathrm{~h}$ of incubation with high production of biomass, and a diminution of $\mathrm{pH}$ until 4.2 (Figure 1B and Supplemental Figure S1; https://doi.org/10.3168/jds.2019-17347). As for traditional yogurt starters, the Lb. bulgaricus strains tested were unable to survive in the medium with galactose as the sole carbon source, whereas the $S$. thermophilus results were strain dependent in the galac- 

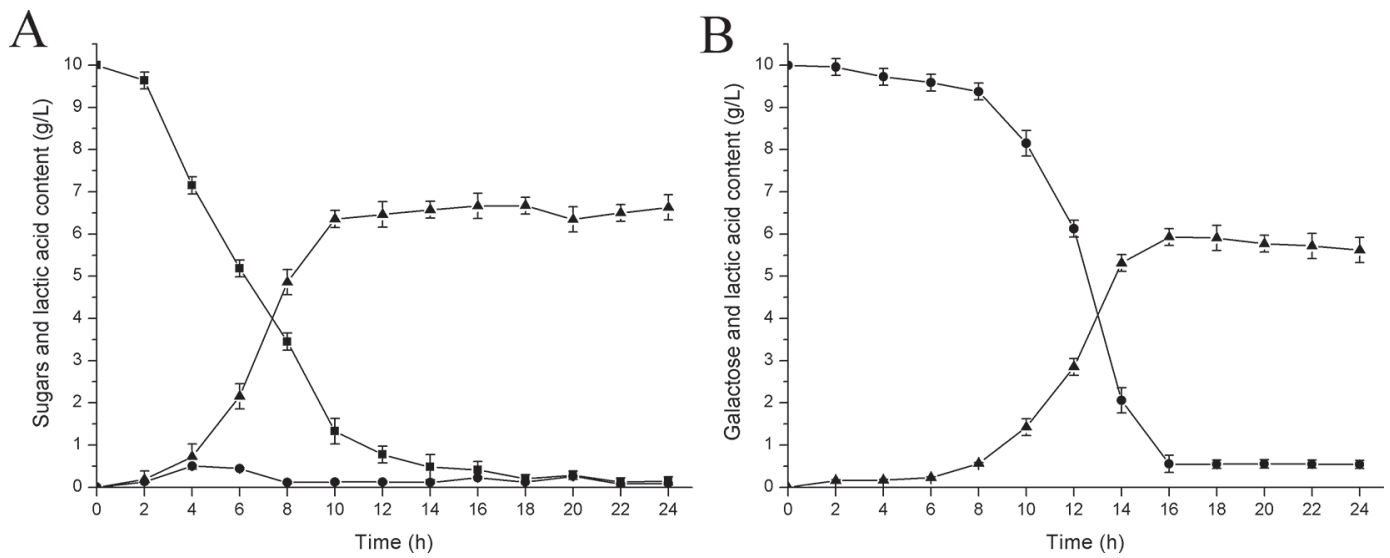

Figure 1. Residual sugars and lactic acid content in de Man, Rogosa, and Sharpe (MRS)-lactose (A) and MRS-galactose (B) inoculated with Lactobacillus plantarum WCFS1. The contents of lactose, galactose, and lactic acid are represented by solid squares, solid circles, and solid triangles, respectively. Error bars represent SE from 3 replicate experiments.

tose utilization. The optical density at $600 \mathrm{~nm}\left(\mathbf{O D}_{600}\right)$ of S. thermophilus CGMCC 7.179 and SDMCC050215 reached values of 1.3 and 0.5 , and the $\mathrm{pH}$ reached values of 5.3 and 5.7, respectively (Supplemental Figure S1; https://doi.org/10.3168/jds.2019-17347). These results indicated that $L b$. plantarum WCFS1 was more capable to use lactose and galactose than $S$. thermophilus and Lb. bulgaricus strains.

\section{Genetic Organization for Lactose/Galactose Metabolism in Lb. plantarum WCFS1}

To investigate the lactose or galactose metabolic flux, we predicted the metabolic pathway by Kyoto Encyclopedia of Genes and Genomes (KEGG database) in $L b$. plantarum WCFS1. As shown in Figure 2A, lactose or galactose was imported through the PTS regulated carbohydrate transporter LacS and RafP. Subsequently, lactose was hydrolyzed by cytosolic $\beta$-galactosidase into glucose and galactose, and these 2 monosaccharides were metabolized by the Embden-Meyerhof-Parnas pathway and Leloir pathway, respectively. In our previous studies, 2 functional $\beta$-galactosidase genes (lacA and lacLM) responsible for lactose hydrolysis were identified in the genome of Lb. plantarum WCFS1 (Zhang et al., 2019). In this study, we found that the genes involved in galactose metabolism were located in the gal operon in the order of galKE $2 \mathrm{~T}$ in Lb. plantarum WCFS1. Among them, galK gene encoded the galactokinase, which is responsible for ATP-dependent phosphorylation of $\alpha$-D-galactose to galactose 1-phosphate. The galT gene encoded the galactose-1-phosphate uridylyltransferase, catalyzing the transfer of a UMP (uridine monophosphate) group from UDP (uridine diphosphate)-glucose to galactose 1-phosphate, thereby generating glucose 1-phosphate and UDP-galactose. The galE gene encoded the UDP-glucose 4-epimerase, responsible for converting UDP-galactose to UDP-glucose to complete the Leloir pathway. In addition, a regulator gene galR was located downstream of the gal operon and was transcribed in the same direction (Figure 2B).

To test the activity of the Leloir pathway in Lb. plantarum WCFS1, the promoter $\mathrm{P}_{\text {gal }}$ of gal operon was cloned and ligated with the GFP reporter gene, generating the recombined plasmid pGal-gfp to explore the characteristics of this promoter. As results, the GFP under gal promoter in $L b$. plantarum WCFS1 could express efficiently in the medium supplemented with glucose, lactose, or galactose as the sole carbon source with fluorescence intensity of 455,651, 476,936, and 369,153, respectively (Figure 3). In addition, compared with the promoter $\mathrm{P}_{\text {ldh }}$ of the lactate dehydrogenase gene $(l d h)$ from $L b$. delbrueckii 11842, which has been proven to be a constitutive promoter for the intracellular production of heterologous proteins in lactobacilli (Sun et al., 2010), the strength of promoter $\mathrm{P}_{\text {gal }}$ was 7- to 9-fold higher than that of promoter $\mathrm{P}_{\text {ldh }}$ (data not shown). These results suggested that the gal operon promoter $\mathrm{P}_{\text {gal }}$ in $L b$. plantarum WCFS1 was a strong constitutive promoter. It was accordance with the above galactose metabolism data of $L b$. plantarum WCFS1, indicating that the gal operon was active in Lb. plantarum WCFS1.

\section{Effects of Lb. plantarum on the Growth and Metabolites of Traditional Starters}

The traditional yogurt starters $S$. thermophilus (CGMCC 7.179 and SDMCC050215) and Lb. bulgaricus (ATCC11842 and SDMCC050201) were adopted to 


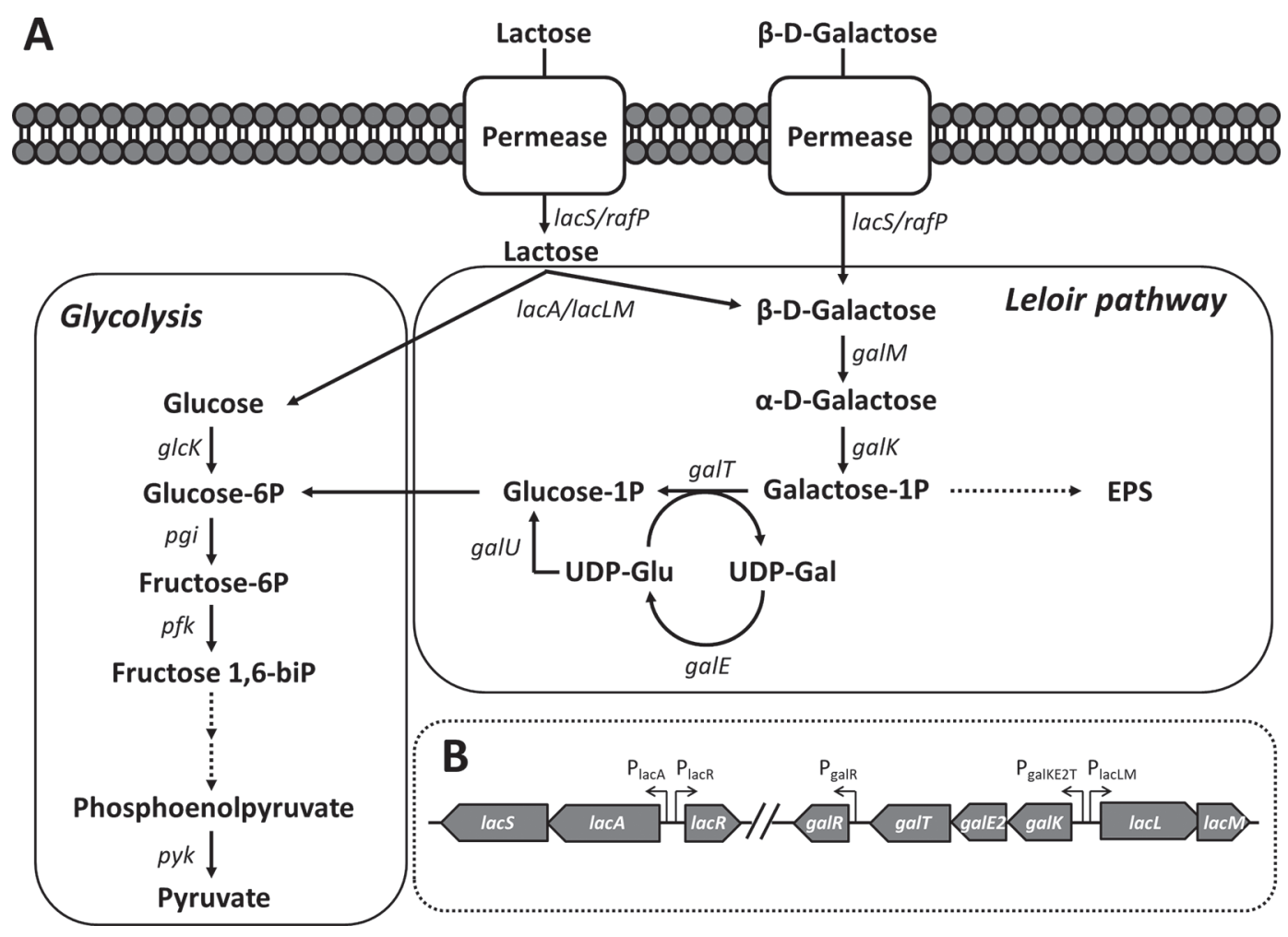

Figure 2. Diagram for lactose and galactose metabolism (A) and genetic organization of lac and gal operon (B) in Lactobacillus plantarum WCFS1. EPS = exopolysaccharides; UDP = uridine diphosphate.

test the growth and metabolism in lactose-based medium with or without $L b$. plantarum WCFS1. When $S$. thermophilus CGMCC 7.179 and SDMCC050215 were cultured alone, the $\mathrm{OD}_{600}$ reached values of 4.2 and 3.8 , and the $\mathrm{pH}$ reached values of 5.3 and 4.9 , respectively.

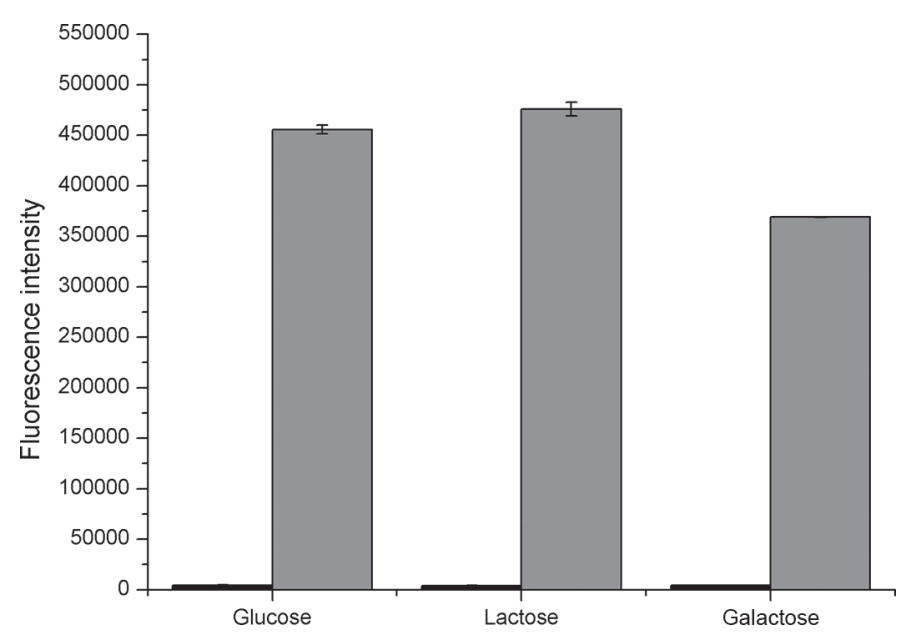

Figure 3. Effects of different carbon sources on the fluorescence intensity of Lactobacillus plantarum WCFS1 (black bar) and WCFS1/ gal-GFP (gray bar). Error bars represent SE from 3 replicate experiments.
When co-cultured with $L b$. plantarum WCFS1, the $\mathrm{OD}_{600}$ increased to 5.0 and 6.0 , and $\mathrm{pH}$ decreased to 4.5 and 4.0, respectively. The same phenomenon was also observed when the strains Lb. bulgaricus ATCC11842 and SDMCC050201 were cultured alone or co-cultured with $L b$. plantarum WCFS1, the $\mathrm{OD}_{600}$ increased from 3.0 and 4.0 to 7.8 , and $\mathrm{pH}$ decreased from 4.8 and 4.5 to 4.2 , respectively (Figure 4). These results indicate that co-cultivation of $L b$. plantarum WCFS1 with traditional starters could increase the total biomass and reduce $\mathrm{pH}$ in lactose-based medium.

The results of viable cell count for each strain are shown in Supplemental Figure S2 (https://doi.org/10 $.3168 /$ jds.2019-17347). The survival of $S$. thermophilus and $L b$. bulgaricus was not damaged in the co-cultivation with $L b$. plantarum WCFS1. In addition, $L b$. plantarum WCFS1 remained at high levels around 9 $\log \mathrm{cfu} / \mathrm{mL}$ in the mixed culture, whereas the viable cells counts of $S$. thermophilus and Lb. bulgaricus were lower compared with $L b$. plantarum WCFS1. In effect, S. thermophilus CGMCC 7.179 reached $8 \log \mathrm{cfu} / \mathrm{mL}$, whereas S. thermophilus SDMCC050215, Lb. bulgaricus ATCC11842 and Lb. bulgaricus SDMCC050201 only reached $7 \log \mathrm{cfu} / \mathrm{mL}$.

The determination of residual sugars and lactic acid content in the lactose-based medium showed that 
A

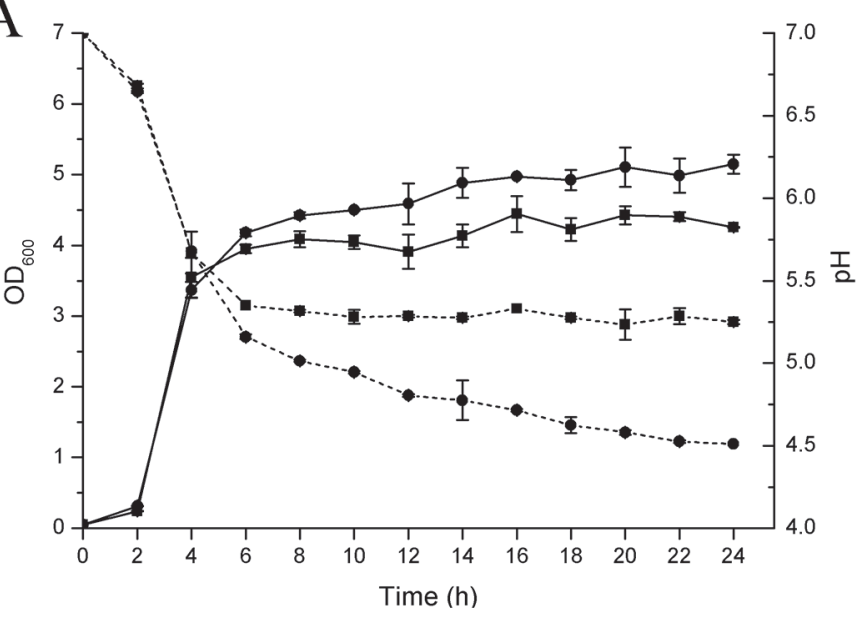

C

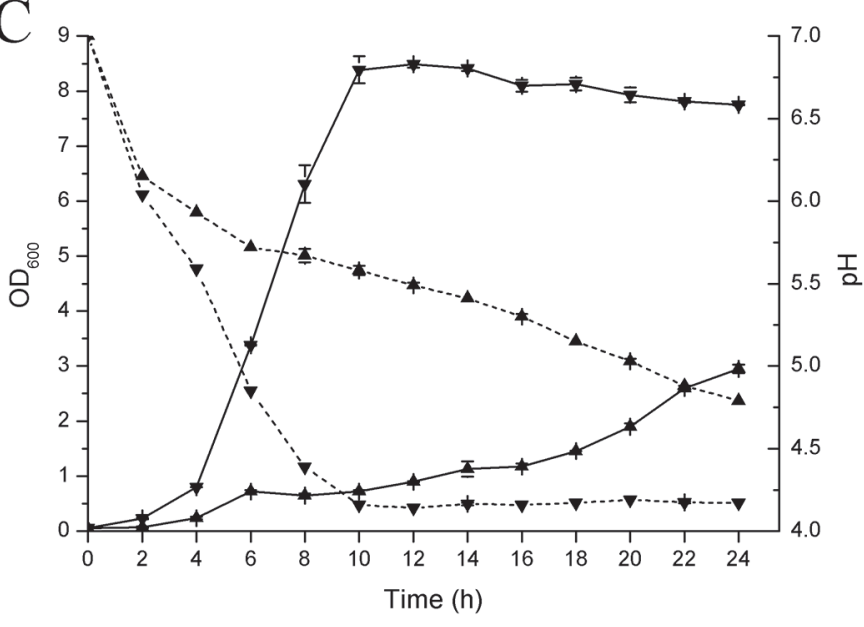

$\mathrm{B}$

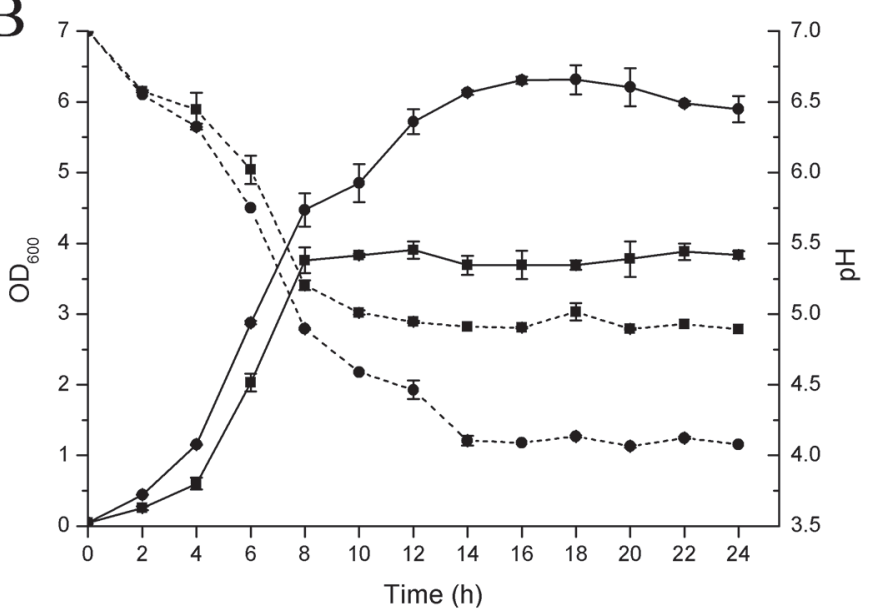

$\mathrm{D}$

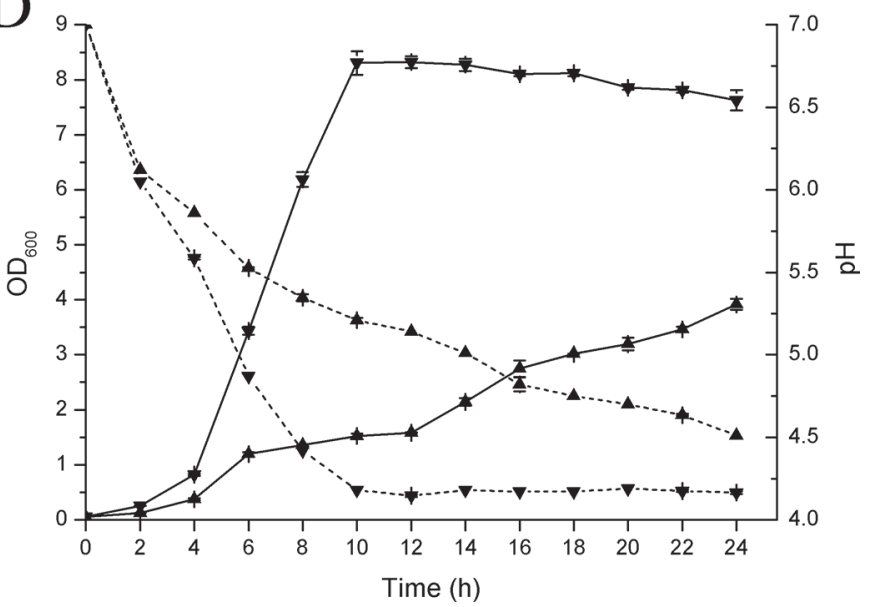

Figure 4. Growth curves and $\mathrm{pH}$ changes of Streptococcus thermophilus CGMCC 7.179 (A), S. thermophilus SDMCC050215 (B), Lactobacillus bulgaricus ATCC11842 (C) and Lb. bulgaricus SDMCC050201 (D) with or without Lb. plantarum WCFS1 in lactose-based medium. Absorbance (optical density at $600 \mathrm{~nm} ; \mathrm{OD}_{600}$ ) and $\mathrm{pH}$ are represented by solid lines and dotted lines, respectively. The culture of $S$. thermophilus or Lb. bulgaricus alone is represented by solid squares or triangles, respectively. The coculture of Lb. plantarum WCFS1 with $S$. thermophilus or Lb. bulgaricus is represented by solid circles or inverted triangles, respectively.

S. thermophilus was more efficient in lactose utilization than Lb. bulgaricus. Streptococcus thermophilus CGMCC 7.179 consumed up $95 \%$ lactose after $6 \mathrm{~h}$ of cultivation, S. thermophilus SDMCC050215 consumed $90 \%$ lactose in the stationary phase, whereas $L b$. bulgaricus ATCC11842 and SDMCC050201 only consumed 77 and $79 \%$ lactose, respectively. In addition, $S$. thermophilus and Lb. bulgaricus accumulated $4 \mathrm{~g} / \mathrm{L}$ to $5 \mathrm{~g} / \mathrm{L}$ galactose in lactose-based medium. Although $S$. thermophilus CGMCC 7.179 was a galactose-positive strain, a large amount of galactose was left in the medium. When they were co-cultured with $L b$. plantarum WCFS1, lactose was consumed more efficiently and galactose also decreased significantly until it was undetectable in the stationary phase. Meanwhile, the content of lactic acid was increased with the decrease of the total sugar content (Figure 5).

\section{Characteristics of Yogurt Fermented by Co-cultivation with Lb. plantarum WCFS1}

To investigate the ability to use lactose or galactose in milk, Lb. plantarum WCFS1 was co-cultured with $S$. thermophilus and Lb. bulgaricus in RSM for $12 \mathrm{~h}$. The residual sugar content of yogurt is shown in Table 1. The initial lactose content in the RSM was 138.77 $\pm 3.61 \mathrm{mmol} / \mathrm{kg}$, and the level of lactose decreased in varying degrees at the end of fermentation. Compared with the yogurt fermented by traditional starters, the cultivation with $L b$. plantarum WCFS1 significantly $(P<0.05$ for all groups) reduced the lactose content. As for galactose content, no significant difference $(P>0.05)$ was observed for SDMCC050201 + SDMCC050215 alone or with WCFS1. In the other 3 combinations, the galactose content increased due the 
A

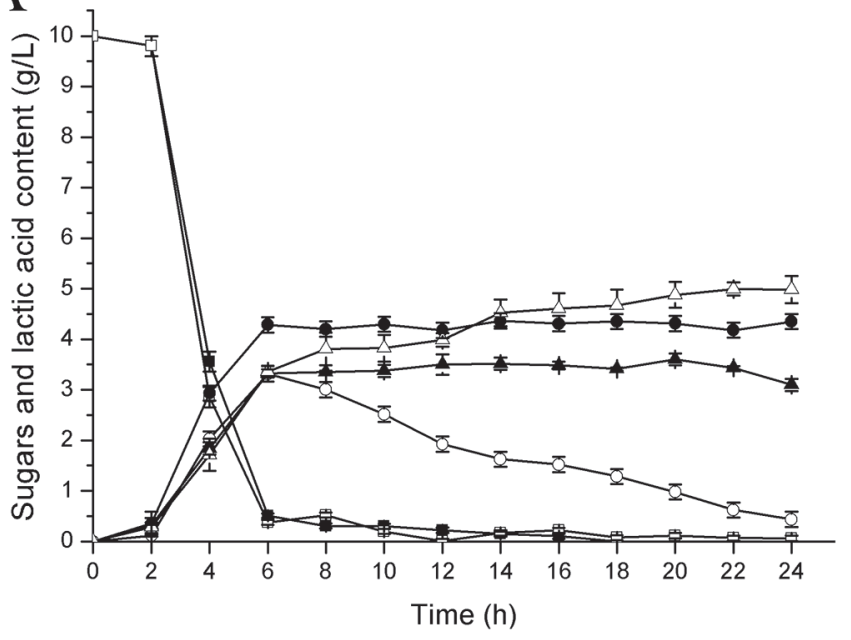

$\mathrm{C}$

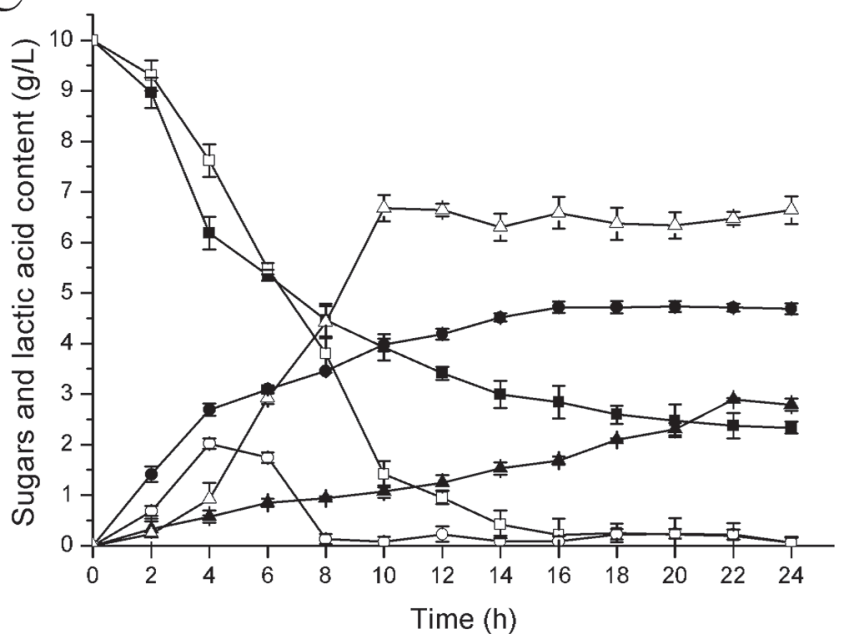

$\mathrm{B}$

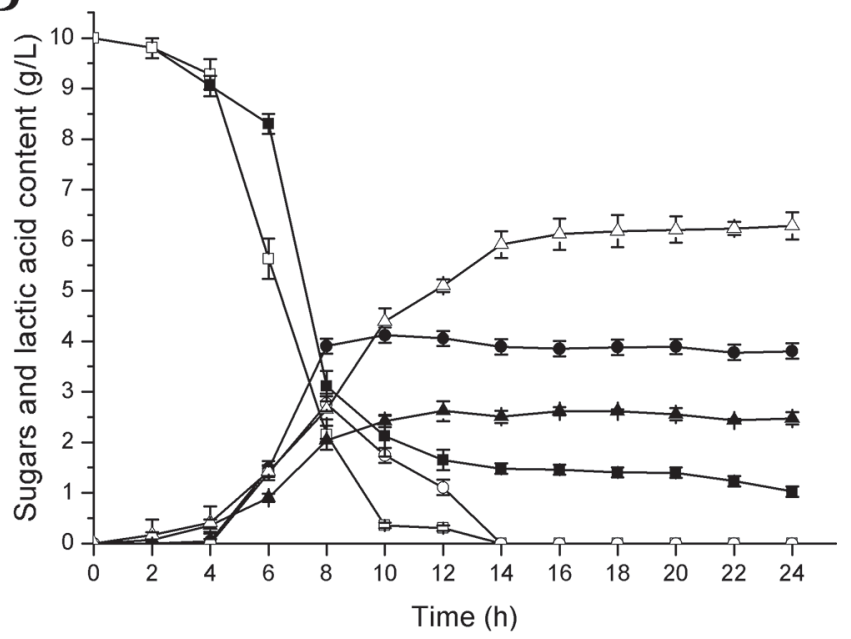

$\mathrm{D}$

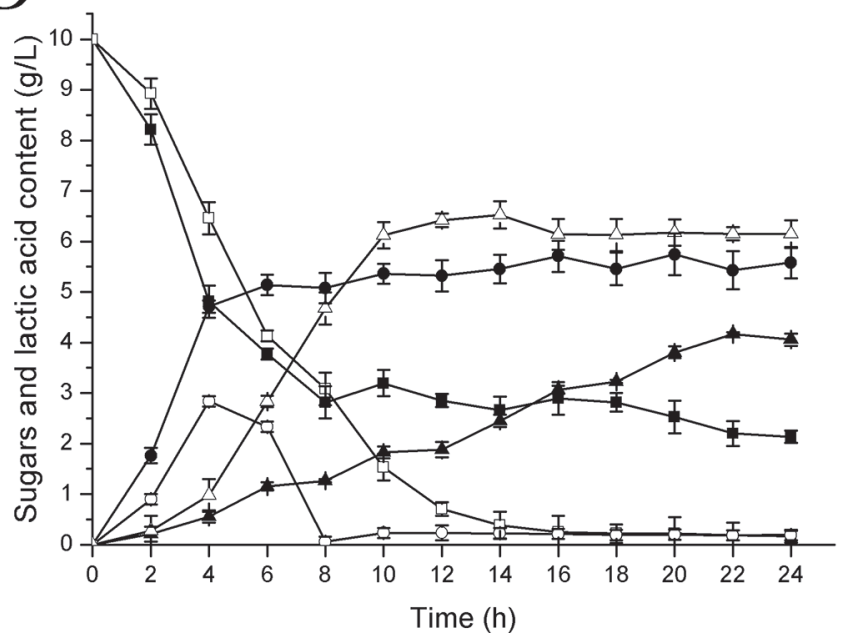

Figure 5. Sugars and lactic acid content in lactose-based medium fermented by Streptococcus thermophilus CGMCC 7.179 (A), S. thermophilus SDMCC050215 (B), Lactobacillus bulgaricus ATCC11842 (C), and Lb. bulgaricus SDMCC050201 (D) with or without Lb. plantarum WCFS1. The results for S. thermophilus or Lb. bulgaricus alone are represented by solid symbols, and the results for the coculture of $L b$. plantarum WCFS1 with $S$. thermophilus or Lb. bulgaricus are represented by open symbols. The contents of lactose, galactose, and lactic acid are represented by squares, circles, and triangles, respectively.

cultivation with $L b$. plantarum WCFS1. The percentages of total sugar reduction, calculated for the molar weight of monosaccharides, are shown in Table 1. Compared with RSM medium, the maximum percentages of total sugar reduction of yogurt fermented by traditional starters and co-cultured with Lb. plantarum WCFS1 were $18.40 \%$ (ATCC11842 + SDMCC050215) and $26.00 \%$ (ATCC11842 + SDMCC050215 + WCFS1) respectively.

The physicochemical properties demonstrated that the cultivation of $L b$. plantarum with traditional yogurt starters could give new characteristics to yogurt (Supplemental Table S2; https://doi.org/10.3168/jds .2019-17347). Compared with the traditional yogurt starters, the co-cultivation with $L b$. plantarum WCFS1 could shorten the curd time significantly $(P<0.05$ for all groups), and the $\mathrm{pH}$ of yogurt decreased and the titratable acidity increased, indicating that the co-cultivation of $L b$. plantarum WCFS1 with traditional starters might improve the yield of lactic acid. In addition, the water-holding capacity was improved for the yogurt fermented by the co-cultivation of $L b$. plantarum WCFS1 with traditional starters. The viable cell counts of Lb. bulgaricus and S. thermophilus in yogurt reached $8 \log \mathrm{cfu} / \mathrm{mL}$, and they were not impaired when co-cultured with $L b$. plantarum WCFS1 during fermentation and storage. In addition, Lb. plantarum WCFS1 also remained at high levels around 8 log cfu/ 
Table 1. Residual sugar content in fermented milks ${ }^{1}$

\begin{tabular}{|c|c|c|c|}
\hline Strain & $\begin{array}{l}\text { Lactose } \\
(\mathrm{mmol} / \mathrm{kg})\end{array}$ & $\begin{array}{l}\text { Galactose } \\
(\mathrm{mmol} / \mathrm{kg})\end{array}$ & $\begin{array}{l}\text { Total sugar } \\
\text { reduction }(\%)\end{array}$ \\
\hline RSM medium & $138.77 \pm 3.61$ & - & - \\
\hline ATCC11842 + CGMCC 7.179 & $116.57 \pm 4.44^{\mathrm{a}}$ & $24.42 \pm 1.11^{\mathrm{b}}$ & 7.20 \\
\hline ATCC11842 + CGMCC $7.179+$ WCFS1 & $94.09 \pm 1.67^{\mathrm{b}}$ & $29.97 \pm 1.67^{\mathrm{a}}$ & 21.40 \\
\hline ATCC11842 + SDMCC050215 & $89.37 \pm 0.56^{\mathrm{a}}$ & $47.74 \pm 1.11^{\mathrm{b}}$ & 18.40 \\
\hline ATCC11842 + SDMCC050215 + WCFS1 & $73.00 \pm 0.56^{\mathrm{b}}$ & $59.39 \pm 0.56^{\mathrm{a}}$ & 26.00 \\
\hline SDMCC050201 + CGMCC 7.179 & $112.13 \pm 1.94^{\mathrm{a}}$ & $22.76 \pm 0.56^{\mathrm{b}}$ & 11.00 \\
\hline SDMCC050201 + CGMCC $7.179+$ WCFS1 & $102.41 \pm 4.16^{\mathrm{b}}$ & $27.20 \pm 1.11^{\mathrm{a}}$ & 16.40 \\
\hline SDMCC050201 + SDMCC050215 & $93.81 \pm 4.16^{\mathrm{a}}$ & $45.52 \pm 2.22^{\mathrm{a}}$ & 16.00 \\
\hline SDMCC050201 + SDMCC050215 + WCFS1 & $81.60 \pm 2.22^{\mathrm{b}}$ & $47.74 \pm 1.67^{\mathrm{a}}$ & 24.00 \\
\hline
\end{tabular}

${ }^{\mathrm{a}, \mathrm{b}}$ Different lowercase superscript letters indicate significant differences $(P<0.05)$ between yogurt fermented by traditional starters with or without Lactobacillus plantarum WCFS1.

${ }^{1}$ Values are presented as mean $\pm \mathrm{SD}$ of 3 individual experiments $(\mathrm{n}=3)$. - indicates data not shown. The percentages of total sugar reduction were calculated by the molar weight of monosaccharide. RSM $=$ reconstituted skim milk.

mL (Supplemental Table S3; https://doi.org/10.3168/ jds.2019-17347).

\section{Sensory Analysis}

Results of the sensory analysis are presented in Supplemental Table S4 (https://doi.org/10.3168/jds .2019-17347). The results showed that co-cultivation of Lb. plantarum WCFS1 with traditional starters did not have a negative effect on the scores of appearance and texture. In contrary, the consumer acceptance of appearance and texture improved significantly $(P<0.05)$ by the cultivation with $L b$. plantarum WCFS1, for the traditional starters ATCC11842 + CGMCC 7.179 and SDMCC050201 + CGMCC 7.179. The flavor scores showed that the effect of co-cultivation with $L b$. plantarum WCFS1 was variable according to the starter used. However, the scores were higher than 8.00 for all groups.

\section{DISCUSSION}

Yogurt making is traditionally performed by the combined actions of $S$. thermophilus and Lb. bulgaricus in milk (Agostoni et al., 2010). During milk fermentation, lactose was efficiently catabolized into glucose and galactose by the $\beta$-galactosidase of $S$. thermophilus and $L b$. bulgaricus, among which the glucose portion was further metabolized by glycolysis. For the galactose portion, the $L b$. bulgaricus strains has not got the genes for their metabolism, and most S. thermophilus strains are unable to ferment galactose despite having intact galKTEM genes encoding the required enzymes for the Leloir pathway (Vaughan et al., 2001; de Vin et al., 2005). The probable reason for this phenotype is due to the defective promoter of gal operon (Vaughan et al., 2001). The shortcoming in the metabolic pathway of $S$. thermophilus and Lb. bulgaricus eventually lead to galactose accumulation as well as residual unfermented lactose in yogurt, which may cause a burden on human health, particularly in individuals with lactose intolerance and galactosemia. Lactobacillus plantarum is encountered as a natural inhabitant of the human gastrointestinal tract with identified candidate probiotic genes and potential health-associated properties (de Vries et al., 2006; Siezen et al., 2012). Several studies were conducted using $L b$. plantarum as a starter for milk fermentation, but most of them focus on its effects on flavor profiles and beneficial effects on intestinal health (Arena et al., 2015; Li et al., 2017). Whether $L b$. plantarum can reduce sugar content in yogurt has not been studied.

The ability of $L b$. plantarum to use lactose and galactose was first detected in artificial media. The results indicated that $L b$. plantarum had a strong ability to metabolize lactose and galactose, and it could catabolize lactose with limited galactose accumulation when lactose is present as the sole carbon. In our previous studies, 2 functional $\beta$-galactosidases responsible for lactose hydrolysis were identified in $L b$. plantarum WCFS1 (Zhang et al., 2019). In this study, the Leloir pathway for galactose metabolism was found to be organized in an operon in this strain. Interestingly, our research also proved that the gal operon was expressed driven by a strong constitutive promoter in Lb. plantarum WCFS1. This property was different from that the sugar utilization is often induced by the corresponding substrate, such as the lactose metabolism in Escherichia coli and the xylose metabolism in Bacillus subtilis (Anbukkarasi et al., 2014b; Chen et al., 2016). Nevertheless, this characteristic of $L b$. plantarum confer it great advantages to use together with traditional starters to reduce sugar content in fermented milk. Because lactose is the main sugar in milk, the MRS-lactose medium was used 
to simulate the milk environment. The same behavior was also observed when $L b$. plantarum WCFS1 was co-cultured with $S$. thermophilus or Lb. bulgaricus. Lactose was consumed more efficiently and galactose also declined significantly until it was undetectable. Meanwhile, the content of lactic acid was increased with the decrease of the total residual sugar content, resulting in a $\mathrm{pH}$ decline, obviously. These results indicated that the co-cultivation of $L b$. plantarum with traditional yogurt starters could contribute to efficient lactose and galactose metabolism and rapid lactic acid production.

Therefore, the milk fermentation was further carried out by $L b$. plantarum WCFS1 coupled with $S$. thermophilus and $L b$. bulgaricus. The results showed that the co-cultivation of $L b$. plantarum WCFS1 could significantly $(P<0.05$ for all groups) reduce the lactose content, which is beneficial for the patients with lactose intolerance. However, the galactose content increased after co-cultivation with $L b$. plantarum WCFS1, which was different from the results in artificial medium. The viable cell count in lactose-based medium showed that Lb. plantarum WCFS1 remained at high levels around 9 $\log \mathrm{cfu} / \mathrm{mL}$, whereas $S$. thermophilus and Lb. bulgaricus only reached 7 to $8 \log \mathrm{cfu} / \mathrm{mL}$. On the contrary, the viable cell counts in fermented milks showed that all strains remained at levels around $8 \log \mathrm{cfu} / \mathrm{mL}$ at the end of the fermentation. These results indicated that Lb. plantarum WCFS1 grew better in lactose-based medium, whereas $S$. thermophilus and Lb. bulgaricus grew better in milk. This could be one of the reasons why the residual galactose content was higher in RSM medium than in lactose-based medium. In addition, the high levels of lactose in milk could mean that the lactose/galactose transporters of $L b$. plantarum WCFS1 prefer to transport lactose rather than galactose in the presence of both lactose and galactose. Therefore, the consumption of extracellular galactose secreted by $S$. thermophilus and Lb. bulgaricus was limited. Compared with RSM medium, the maximum sugar reductions for yogurts with and without $L b$. plantarum were 18.40 and $26.00 \%$, respectively. The molar quantity of the accumulated galactose was less than the molar quantity of the hydrolyzed lactose, indicating that a certain amount of galactose was metabolized by $L b$. plantarum. Hence, co-cultivation of $L b$. plantarum with traditional yogurt starters could reduce the total sugar content in yogurt.

The physicochemical characteristics demonstrated that co-cultivation of $L b$. plantarum with traditional yogurt starters in yogurt making had several advantages. First, the curd time was shortened, which will increase production efficiency and promote the economic benefits of enterprises. Second, the syneresis was reduced significantly $(P<0.05$ for all groups $)$ compared with traditional fermented milk, which may be related to the content of casein, whey protein, and exopolysaccharide (Hassan et al., 2003; Meletharayil et al., 2018). Considering syneresis (wheying off) is a major defect in the yogurt industry and is directly related to the extent of physical disturbance to the network of protein micelles, stabilizers such as pectin, gelatin, and starch are often used in yogurt making to prevent wheying off (Tasneem et al., 2014). Hence, the use of Lb. plantarum would reduce the cost of the product. Third, sensory analysis indicated that yogurt fermented by $L b$. plantarum WCFS1 with traditional starters was acceptable to consumers in appearance, texture, and flavor. Above all, there is great development potential to prepare lowsugar yogurt by co-cultivation of $L b$. plantarum with traditional yogurt starters.

\section{ACKNOWLEDGMENTS}

This work was supported by a grant from the National Natural Science Foundation of China (31871767, Beijing), National Key Research and Development Program of China (2017YFD0400300, Beijing), and Public Service Sectors (Agriculture) Special and Scientific Research Projects (201503134, Beijing, China). The authors declare no conflicts of interest.

\section{REFERENCES}

Agostoni, C., J. L. Bresson, S. Fairweather-Tait, A. Flynn, I. Golly, H. Korhonen, P. Lagiou, M. Løvik, R. Marchelli, A. Martin, B. Moseley, M. Neuhäuser-Berthold, H. Przyrembel, S. Salminen, Y. Sanz, S. Strain, S. Strobel, I. Tetens, D. Tomé, H. van Loveren, and H. Verhagen. 2010. Scientific opinion on the substantiation of health claims related to live yoghurt cultures and improved lactose digestion (ID 1143, 2976) pursuant to article 13(1) of regulation (EC) No 1924/2006. EFSA J. 8:1763. https://doi.org/10.2903/j .efsa.2010.1763.

Anbukkarasi, K., D. K. Nanda, T. UmaMaheswari, T. Hemalatha, P. Singh, and R. Singh. 2014b. Assessment of expression of Leloir pathway genes in wild-type galactose-fermenting Streptococcus thermophilus by real-time PCR. Eur. Food Res. Technol. 239:895903. https://doi.org/10.1007/s00217-014-2286-9.

Anbukkarasi, K., T. UmaMaheswari, T. Hemalatha, D. K. Nanda, P. Singh, and R. Singh. 2014a. Preparation of low galactose yogurt using cultures of Gal $(+)$ Streptococcus thermophilus in combination with Lactobacillus delbrueckii ssp. bulgaricus. J. Food Sci. Technol. 51:2183-2189. https://doi.org/10.1007/s13197-014-1262-5.

Arena, M. P., G. Caggianiello, P. Russo, M. Albenzio, S. Massa, D. Fiocco, V. Capozzi, and G. Spano. 2015. Functional starters for functional yogurt. Foods 4:15-33. https://doi.org/10.3390/ foods 4010015 .

Aryana, K. J., and D. W. Olson. 2017. A 100-year review: Yogurt and other cultured dairy products. J. Dairy Sci. 100:9987-10013. https: //doi.org/10.3168/jds.2017-12981.

Benozzi, E., A. Romano, V. Capozzi, S. Makhoul, L. Cappellin, I. Khomenko, E. Aprea, M. Scampicchio, G. Spano, T. D. Märk, F. Gasperi, and F. Biasioli. 2015. Monitoring of lactic fermentation driven by different starter cultures via direct injection mass spectrometric analysis of flavour-related volatile compounds. Food Res. Int. 76:682-688. https://doi.org/10.1016/j.foodres.2015.07 .043 . 
Capozzi, V., M. Fragasso, R. Romaniello, C. Berbegal, P. Russo, and G. Spano. 2017. Spontaneous food fermentations and potential risks for human health. Fermentation 3:49. https://doi.org/10 .3390 /fermentation3040049.

Chen, J., Y. Zhu, G. Fu, Y. Song, Z. Jin, Y. Sun, and D. Zhang. 2016. High-level intra- and extra-cellular production of D-psicose 3-epimerase via a modified xylose-inducible expression system in Bacillus subtilis. J. Ind. Microbiol. Biotechnol. 43:1577-1591. https://doi.org/10.1007/s10295-016-1819-6.

Chiu, C. H., T. Y. Lu, Y. Y. Tseng, and T. M. Pan. 2006. The effects of Lactobacillus-fermented milk on lipid metabolism in hamsters fed on high-cholesterol diet. Appl. Microbiol. Biotechnol. 71:238 245. https://doi.org/10.1007/s00253-005-0145-0.

Chollet, M., D. Gille, A. Schmid, B. Walther, and P. Piccinali. 2013. Acceptance of sugar reduction in flavored yogurt. J. Dairy Sci. 96:5501-5511. https://doi.org/10.3168/jds.2013-6610.

da Costa, M. P., S. Frasao Bda, B. R. Lima, B. L. Rodrigues, and C. A. Conte Junior.. 2016. Simultaneous analysis of carbohydrates and organic acids by HPLC-DAD-RI for monitoring goat's milk yogurts fermentation. Talanta 152:162-170. https://doi.org/10 .1016/j.talanta.2016.01.061.

de Vin, F., P. Rådstrom, L. Herman, and L. De Vuyst. 2005. Molecular and biochemical analysis of the galactose phenotype of dairy Streptococcus thermophilus strains reveals four different fermentation profiles. Appl. Environ. Microbiol. 71:3659-3667. https://doi.org/ 10.1128/AEM.71.7.3659-3667.2005.

de Vries, M. C., E. E. Vaughan, M. Kleerebezem, and W. M. de Vos. 2006. Lactobacillus plantarum: Survival, functional and potential probiotic properties in the human intestinal tract. Int. Dairy J. 16:1018-1028. https://doi.org/10.1016/j.idairyj.2005.09.003.

Harju, M., H. Kallioinen, and O. Tossavainen. 2012. Lactose hydrolysis and other conversions in dairy products: technological aspects. Int. Dairy J. 22:104-109. https://doi.org/10.1016/j.idairyj.2011.09 .011 .

Hassan, A. N., R. Ipsen, T. Janzen, and K. B. Qvist. 2003. Microstructure and rheology of yogurt made with cultures differing only in their ability to produce exopolysaccharides. J. Dairy Sci. 86:16321638. https://doi.org/10.3168/jds.S0022-0302(03)73748-5.

Hols, P., F. Hancy, L. Fontaine, B. Grossiord, D. Prozzi, N. LeblondBourget, B. Decaris, A. Bolotin, C. Delorme, S. D. Ehrlich, E. Guédon, V. Monnet, P. Renault, and M. Kleerebezem. 2005. New insights in the molecular biology and physiology of Streptococcus thermophilus revealed by comparative genomics. FEMS Microbiol. Rev. 29:435-463. https://doi.org/10.1016/j.femsre.2005.04.008.

Horiuchi, H., N. Inoue, E. Liu, M. Fukui, Y. Sasaki, and T. Sasaki. 2009. A method for manufacturing superior set yogurt under reduced oxygen conditions. J. Dairy Sci. 92:4112-4121. https://doi .org/10.3168/jds.2008-1747.

Jelen, P., and O. Tossavainen. 2003. Low lactose and lactose-free milk and dairy products-prospects, technologies and applications. Aust. J. Dairy Technol. 58:161-165.

Li, C., J. Song, L. Y. Kwok, J. Wang, Y. Dong, H. Yu, Q. Hou, H. Zhang, and Y. Chen. 2017. Influence of Lactobacillus plantarum on yogurt fermentation properties and subsequent changes during postfermentation storage. J. Dairy Sci. 100:2512-2525. https://doi .org/10.3168/jds.2016-11864.

Marco, M. L., D. Heeney, S. Binda, C. J. Cifelli, P. D. Cotter, B. Foligné, M. Gänzle, R. Kort, G. Pasin, A. Pihlanto, E. J. Smid, and R. Hutkins. 2017. Health benefits of fermented foods: Microbiota and beyond. Curr. Opin. Biotechnol. 44:94-102. https://doi.org/ 10.1016/j.copbio.2016.11.010.

Meletharayil, G. H., H. A. Patel, L. E. Metzger, C. Marella, and T. Huppertz. 2018. Influence of partially demineralized milk proteins on rheological properties and microstructure of acid gels. J. Dairy Sci. 101:1864-1871. https://doi.org/10.3168/jds.2017-13670.
Neves, A. R., W. A. Pool, A. Solopova, J. Kok, H. Santos, and O. P. Kuipers. 2010. Towards enhanced galactose utilization by Lactococcus lactis. Appl. Environ. Microbiol. 76:7048-7060. https://doi .org/10.1128/AEM.01195-10.

Robitaille, G., S. Moineau, D. St-Gelais, C. Vadeboncoeur, and M. Britten. 2007. Galactose metabolism and capsule formation in a recombinant strain of Streptococcus thermophilus with a galactosefermenting phenotype. J. Dairy Sci. 90:4051-4057. https://doi .org/10.3168/jds.2007-0140.

Siezen, R. J., C. Francke, B. Renckens, J. Boekhorst, M. Wels, M. Kleerebezem, and S. A. van Hijum. 2012. Complete resequencing and reannotation of the Lactobacillus plantarum WCFS1 genome. J. Bacteriol. 194:195-196. https://doi.org/10.1128/JB.06275-11.

Silanikove, N., G. Leitner, and U. Merin. 2015. The interrelationships between lactose intolerance and the modern dairy industry: Global perspectives in evolutional and historical backgrounds. Nutrients 7:7312-7331. https://doi.org/10.3390/nu7095340.

Sun, Z., J. Kong, and W. Kong. 2010. Characterization of a cryptic plasmid pD403 from Lactobacillus plantarum and construction of shuttle vectors based on its replicon. Mol. Biotechnol. 45:24-33. https://doi.org/10.1007/s12033-010-9242-0.

Tasneem, M., F. Siddique, A. Ahmad, and U. Farooq. 2014. Stabilizers: Indispensable substances in dairy products of high rheology. Crit. Rev. Food Sci. Nutr. 54:869-879. https://doi.org/10.1080/ 10408398.2011.614702.

Turner, K. W., and F. G. Martley. 1983. Galactose fermentation and classification of thermophilic lactobacilli. Appl. Environ. Microbiol. 45:1932-1934. https://doi.org/10.1128/AEM.45.6.1932-1934.1983.

Valente, G. L. C., L. B. Acurcio, L. P. V. Freitas, J. R. Nicoli, A. M. Silva, M. R. Souza, and C. F. A. M. Penna. 2019. Short communication: In vitro and in vivo probiotic potential of Lactobacillus plantarum B7 and Lactobacillus rhamnosus D1 isolated from Minas artisanal cheese. J. Dairy Sci. 102:5957-5961. https://doi .org/10.3168/jds.2018-15938.

Van Calcar, S. C., L. E. Bernstein, F. J. Rohr, C. H. Scaman, S. Yannicelli, and G. T. Berry. 2014. A re-evaluation of life-long severe galactose restriction for the nutrition management of classic galactosemia. Mol. Genet. Metab. 112:191-197. https://doi.org/10 .1016/j.ymgme.2014.04.004.

Vaughan, E. E., P. T. van den Bogaard, P. Catzeddu, O. P. Kuipers, and W. M. de Vos. 2001. Activation of silent gal genes in the lacgal regulon of Streptococcus thermophilus. J. Bacteriol. 183:11841194. https://doi.org/10.1128/JB.183.4.1184-1194.2001.

Wu, Q., C. K. W. Cheung, and N. P. Shah. 2015. Towards galactose accumulation in dairy foods fermented by conventional starter cultures: Challenges and strategies. Trends Food Sci. Technol. 41:2436. https://doi.org/10.1016/j.tifs.2014.08.010.

Xu, Z., H. He, S. Zhang, and J. Kong. 2017. Effects of inoculants Lactobacillus brevis and Lactobacillus parafarraginis on the fermentation characteristics and microbial communities of corn stover silage. Sci. Rep. 7:13614. https://doi.org/10.1038/s41598-017-14052 -1 .

Xu, Z., S. Zhang, Y. Mu, and J. Kong. 2018. Paenibacillus panacisoli enhances growth of Lactobacillus spp. by producing xylooligosaccharides in corn stover ensilages. Carbohydr. Polym. 184:435-444. https://doi.org/10.1016/j.carbpol.2017.12.044.

Zhang, S., Z. Xu, L. Qin, and J. Kong. 2019. Development of strong lactose/galactose-inducible expression system for Lactobacillus plantarum by optimizing promoter. Biochem. Eng. J. 151:107316. https://doi.org/10.1016/j.bej.2019.107316.

\section{ORCIDS}

J. Kong ๑ https://orcid.org/0000-0002-2872-2078 